\title{
Erratum: Mass spectrometry and proteomics: hitting the mark
}

\section{Nathan Blow}

Nat. Methods 5, 741-747 (2008); published online 1 August 08; corrected after print 29 September 2008

In the version of this article initially published, Box 1 incorrectly listed the University of California, Berkeley as one of the five Clinical Proteomic Technology Assessment for Cancer (CPTAC) groups. The University of California, San Francisco-Lawrence Berkeley National Laboratory-Buck Institute is the correct designation for this CPTAC group. This error has been corrected in the HTML and PDF versions of the article. 